\title{
ELECTRICAL THROUGH WAFER INTERCONNECTS WITH 0.05 PICO FARADS PARASITIC CAPACITANCE ON $400 \mu M$ THICK SILICON SUBSTRATE
}

\author{
Ching H. Cheng, Arif S. Ergun, and Butrus T. Khuri-Yakub \\ Edward L. Ginzton Laboratory, Stanford University \\ Stanford, CA 94305-4085
}

\begin{abstract}
This paper presents a technology for high density and low parasitic capacitance electrical through-wafer interconnects (vias) to an array of micromachined transducers on a silicon wafer. Vertical wafer feedthroughs (interconnects) connect an array of sensors or actuators from the front side (transducer side) to the backside (packaging side) of the wafer. A 20 to 1 high aspect ratio $400 \mu \mathrm{m}$ long and $20 \mu \mathrm{m}$ diameter interconnect is achieved by using deep reactive ion etching (DRIE). Reduction of the parasitic capacitance to the substrate is achieved using reverse-biased pnjunction diodes operating in the depletion region. A parasitic capacitance of $0.05 \mathrm{pF}$ has been demonstrated by this approach. This three-dimensional architecture allows for elegant wafer-level packaging through simple flip-chip bonding of the chip's backside to a printed circuit board (PCB) or a signal processing wafer.
\end{abstract}

\section{INTRODUCTION}

In micro-electro-mechanical-systems (MEMS) applications, it is advantageous to have electronic circuitry as near the sensor/actuator as possible. However, integrating both the MEMS devices with electronics on the same wafer often leads to a compromise between the performance of either or both systems. An excellent solution to this problem is to construct the optimum MEMS devices and electronics on separate wafers, provide a through wafer interconnect with minimum resistance and capacitance on the MEMS wafer, then flip-chip bond the two wafers (Fig. 1). In this fashion, the MEMS wafer can be fully populated such as in applications of infra-red (IR) focal plane arrays or three-dimensional ultrasound imaging [1-5]. Finally, since the MEMS and electronics wafers can be fabricated in different facilities, the overall yield of the manufacturing process is enhanced.

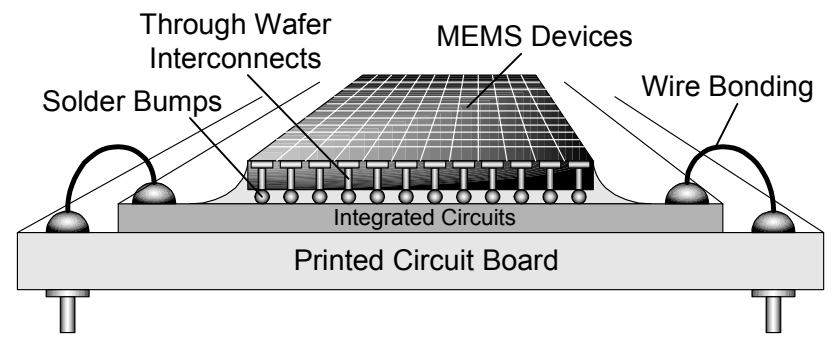

Figure 1. Packaging schematic of through-wafer interconnects.

The architecture of achieving this hybrid integration with high density is based on through-wafer vertical interconnects with high aspect ratio. Many processes have been previously used to fabricate through-wafer interconnects [6][7] including dry etched polysilicon filled interconnects by Chow et al [8]. In previous work, we integrated similar interconnects into an active sensor arrays and made improvements on parasitic capacitance from $2 \mathrm{pF}$ to $0.28 \mathrm{pF}[\mathbf{1}]$.
To further reduce the parasitic capacitance, we propose to use a through wafer interconnect as shown in Fig. 2. The interconnect presents a parallel capacitance and a series resistance to the input impedance of the MEMS device. Thus, for operation that is not limited by the interconnect, both the capacitance and resistance have to be very small. We will present a technology where the parasitic capacitance is reduced to $0.05 \mathrm{pF}$ in a silicon wafer that is $400 \mu \mathrm{m}$ thick with a resistivity of $1000 \Omega-\mathrm{cm}$ and with a via that has a diameter of $20 \mu \mathrm{m}$.

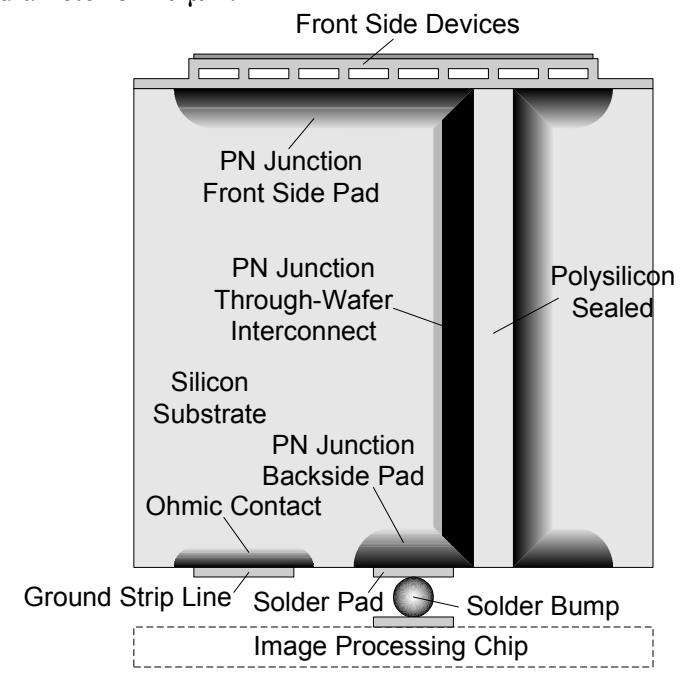

Figure 2. Schematic of the through-wafer interconnect.

For our current application, the through-wafer interconnect is used for integration of two-dimensional ultrasonic transducer arrays with electronic dies. In an ultrasonic transducer array operation, the parasitic capacitance of the interconnect between an array element and its transmit/receive electronics is the limiting factor for the dynamic range and bandwidth. Therefore, it is always best to put the electronics as close to the array elements as possible. In this work, we demonstrate a method to integrate a 128 $\mathrm{x} 128$ capacitive micromachined ultrasonic transducer (CMUT) array with the electronic circuit without sacrificing the performance of either while minimizing the parasitic capacitance. To do this, an electrical through-wafer interconnect is employed to address the array elements individually, where the front side of the wafer is fully populated with the ultrasonic array elements, and the backside is solely dedicated to bond pads for the flip-chip bonding to the printed circuit board (PCB) or the integrated circuits (Fig. 1). In this manner, the parasitics due to any interconnection cable are avoided. To further improve the device performance, the parasitic capacitance of the through-wafer interconnects to the silicon substrate needs to be reduced to a much lower level than the device capacitance.

Travel support has been generously provided by the Transducers Research Foundation and by the DARPA MEMS and DARPA bioFlips Programs. 


\section{PARASITIC CAPACITANCE REDUCTION}

The via can either have a metal-insulator-semiconductor (MIS) or a PN junction diode relationship with the silicon. In order to reduce the capacitance of the via, it is best to have a reversed biased pn junction because the depletion region can be made very large, as shown in Fig. 3. Hence, the parasitic capacitance can be reduced to about $0.05 \mathrm{pF}$. Two-sided deep reactive ion etching (RIE) is used to make through wafer holes with an aspect ration of 20:1. In previous work [1], we made vias with MIS junctions that had a parasitic capacitance of $0.28 \mathrm{pF}$. However, in this work, we report vias with PN junction diodes that have a measured parasitic capacitance of $0.05 \mathrm{pF}$ at a reverse bias more than $10 \mathrm{~V}$ as seen in Fig. 11. This will allow operation of MEMS devices at very high frequencies and without a loss of dynamic range due to the interconnect. The parasitic capacitance can be further reduced by reducing the thickness of the wafer, the diameter of the via, and increasing the resistivity of the silicon wafer.

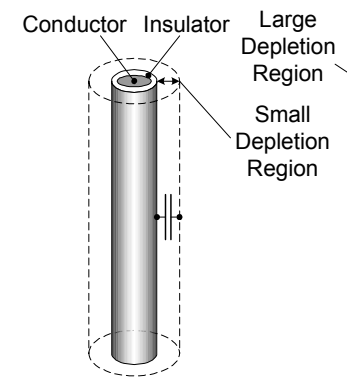

a. Isolation with insulator

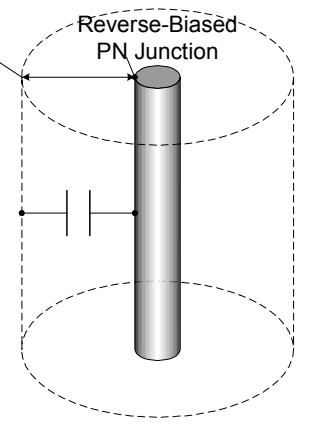

b. Isolation with back-to-back reverse-biased pn junction
Figure 3. Schematic of the MIS and PN junctions.

Parasitic capacitance has a detrimental effect on the performance of the CMUT. For each array element, there are three sources contributing to the parasitic capacitance: the front side 400 $\mu \mathrm{m} \times 400 \mu \mathrm{m}$ pad for the bottom electrode of the transducers; the backside $200 \mu \mathrm{m} \times 140 \mu \mathrm{m}$ pad for bonding; and the throughwafer interconnect with $400 \mu \mathrm{m}$ length and $20 \mu \mathrm{m}$ diameter (Fig. 4). The optimum solution for parasitic capacitance reduction is to implement reverse-biased pn-junction diodes on the front and backside pads of the wafer and inside the interconnects as shown in Fig. 3. When the pn junction is reversed biased (DC), the high resistivity $(>1000 \mathrm{ohm}-\mathrm{cm})$ silicon substrate is fully depleted, thus a low parasitic capacitance is achieved.

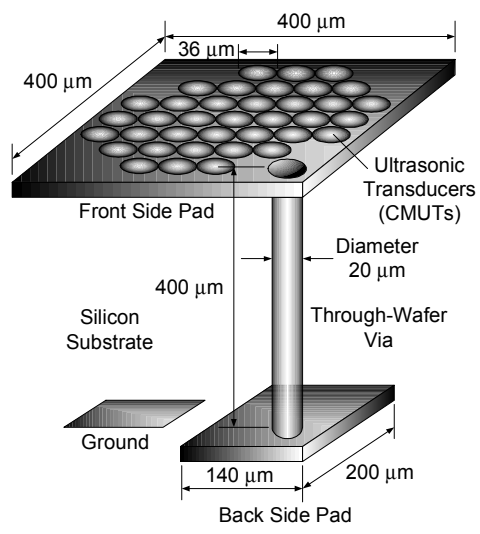

Figure 4. Schematic of the MIS and PN junctions.

\section{EXPECTED PARASITICS}

Based on simulation, a silicon substrate with resistivity of $1000 \mathrm{ohm}-\mathrm{cm}$ is used. A reversed bias voltage is applied to drive the pn junction diodes into the depletion region. The expected capacitance-voltage $(\mathrm{CV})$ relationship is shown in Figure 5. We expect the total parasitic capacitance to be lower than $0.06 \mathrm{pF}$ for a reverse bias voltage of more than 10 volts. This includes capacitance of the front and backside pads and a single throughwafer interconnect, which is a substantial improvement over previously reported results [1]. The predicted series resistance is $434 \Omega$ which assumes that the doping profile is the same for the surfaces on top of the wafer and inside the via holes.

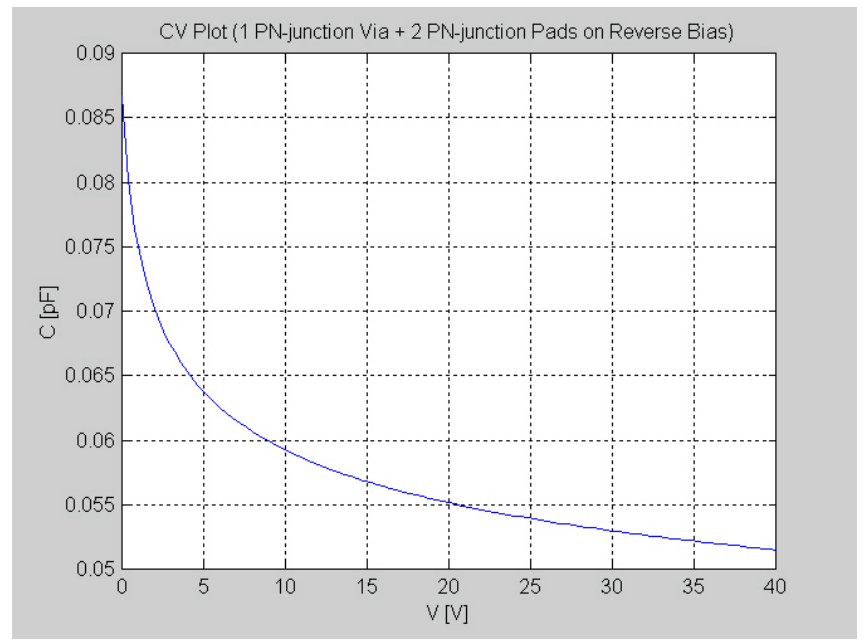

Figure 5. Expected parasitic capacitance

\section{THROUGH WAFER INTERCONNECT PROCESS}

The process flow is shown in Fig. 6. We start with a $400 \mu \mathrm{m}$ thick double-sided polished n-type $<100>\mathrm{Si}$ wafer which is thermally oxidized to $2 \mu \mathrm{m}$ thick to serve as a hard mask for the deep etch. Both sides are then patterned with $20 \mu \mathrm{m}$ diameter openings for each interconnect (Fig. 6a). The through-wafer deep etch is done by etching half way from both sides of the wafer (Fig. 6b). By this means, a 20 to 1 high aspect ratio via hole can be achieved. When the interconnect is etched through, the helium flow used for cooling goes through the etched holes, and the etching will be slowed down, serving as an etch stop. The wafer is then heavily doped with boron to build the pn junction diode inside the holes (Fig. 6c). The interconnect holes are then filled with polysilicon (Fig. 6d). The polysilicon on both sides is then etched back and stopped on the oxide (Fig. 6e). It is ready to be etched for the front and back side oxide opening (Fig. 6f). The wafer is then doped with boron which makes up the pn junctions for the front and backside pads. The oxide is etched and the backside is patterned with photoresist for phosphorous ion implantation for ohmic contact (Fig. 6g). After this step, the transducers can be built on top of the front side pn-junction pad (Fig. 8). At the very end, the backside metal pads for flip-chip bonding are formed by lift-off (Fig. 6h). The SEM pictures show the cross section of a finished interconnect in Fig. 7. The wafer is ready for flip-chip bonding to a circuit chip or PCB (Fig. 9). 


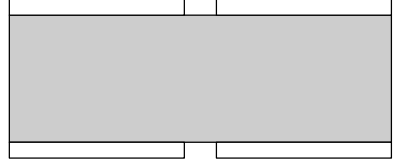

b) Deep Etch

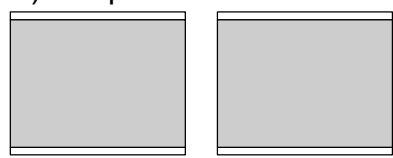

c) Via Diffusion

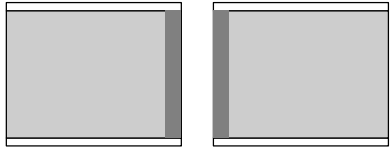

d) Poly Seal
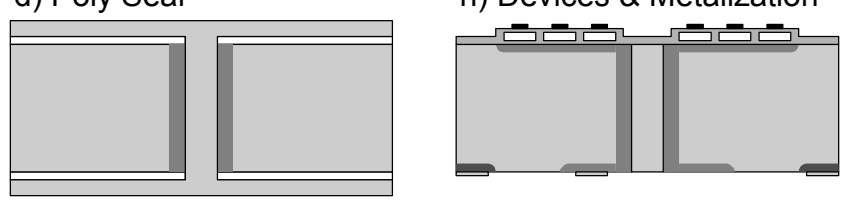

Figure 6. Fabrication process for through wafer interconnects.

a)

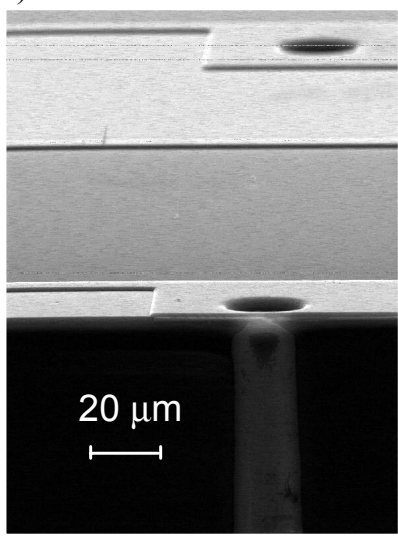

b)

Figure 7. SEM pictures of the cross section of the interconnects.

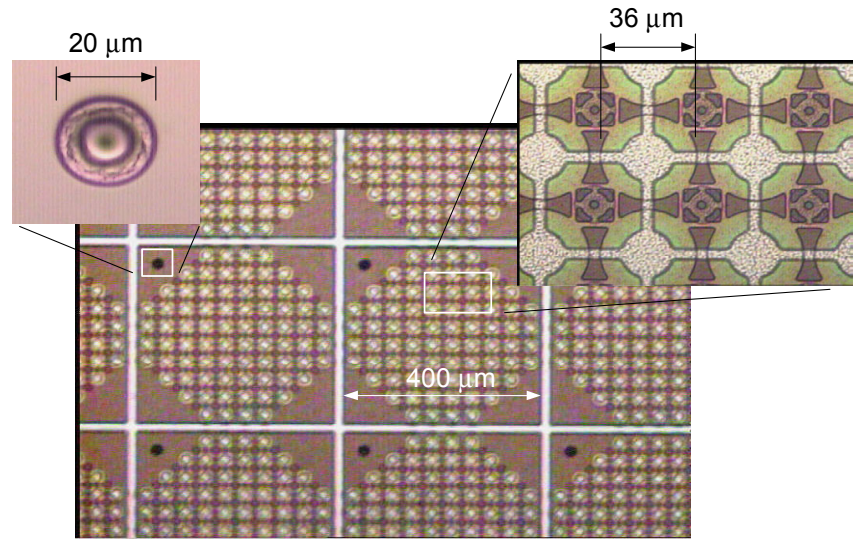

Figure 8. Photographs of CMUT array elements with through wafer interconnects.

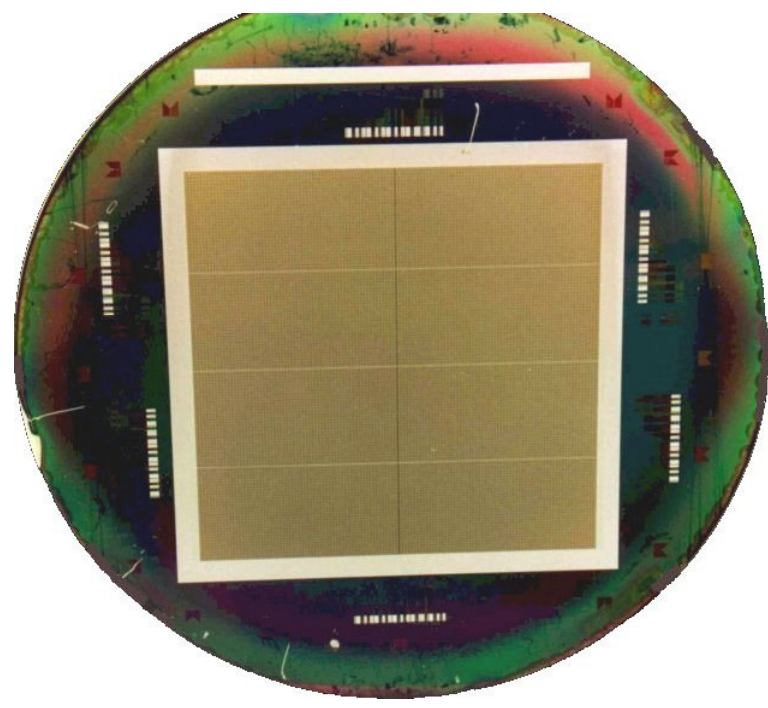

Figure 9. Photograph of a finished $128 \times 128$ CMUT array on a 4 " silicon wafer.

\section{TEST RESULTS}

As shown in Fig. 10, a testing device with a through-wafer interconnect connected with both front side and back side pads and a ground to the substrate is employed to measure the $\mathrm{C}-\mathrm{V}$ characteristic at $1 \mathrm{MHz}$ frequency. A reversed DC bias is applied to drive both pn-junction pads into the depletion region. The total capacitance is the capacitance of the through-wafer interconnect plus both pads. The series resistance for each via is $900 \Omega$ which is more than our prediction of $434 \Omega$. This discrepancy comes from the non-uniform doping profile throughout the via hole. However, we assumed a uniform doping profile for our calculation. Compared with the top wafer surface, there is less dopant getting into the via hole. The doping profile inside the via hole depends on the uniformity during the boron diffusion process which is hard to achieve without a special simulation tool [9]. The leakage current coming from both pads and the via is $7 \mathrm{nA}$.

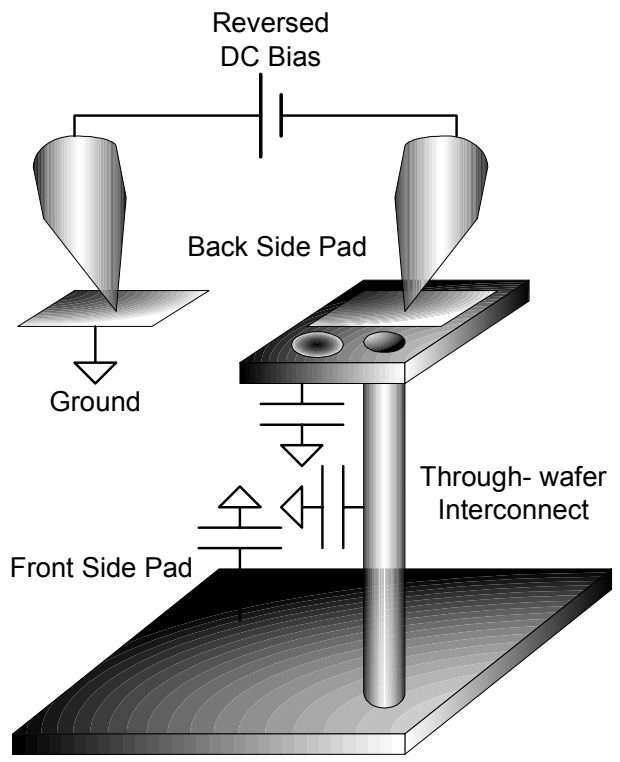

Figure 10. Capacitance measurement set-up. 
Fig. 11 shows a $\mathrm{C}-\mathrm{V}$ characteristics at $1 \mathrm{MHz}$ frequency. By applying a reverse bias for more than 10 volts the capacitance will decrease to $0.055 \mathrm{pF}$ because of depletion into the substrate. This experimental result is very close to what we expected from the simulation (Fig. 12).

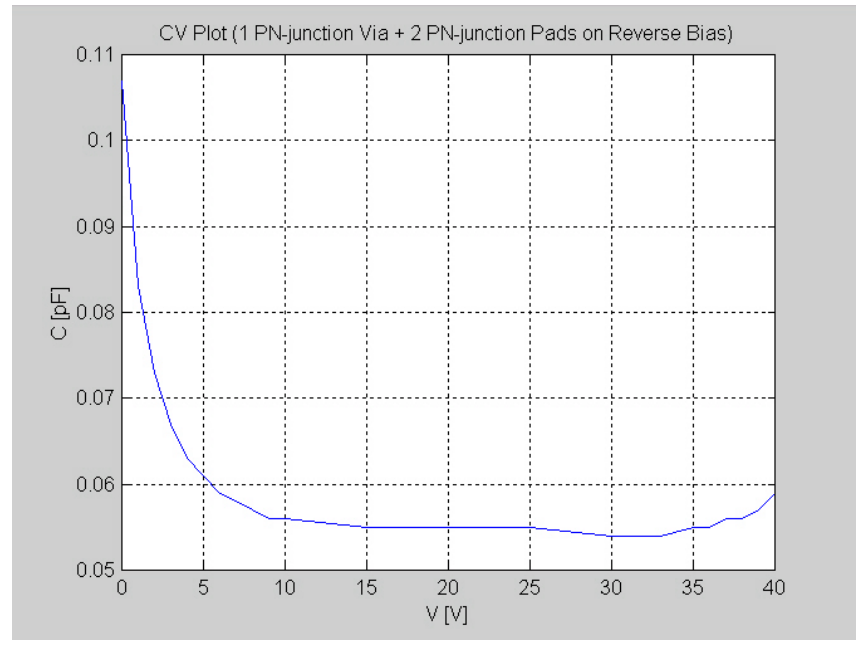

Figure 11. Measured capacitance-voltage relationship (CV) of a reversed biased pn junction through wafer interconnect.

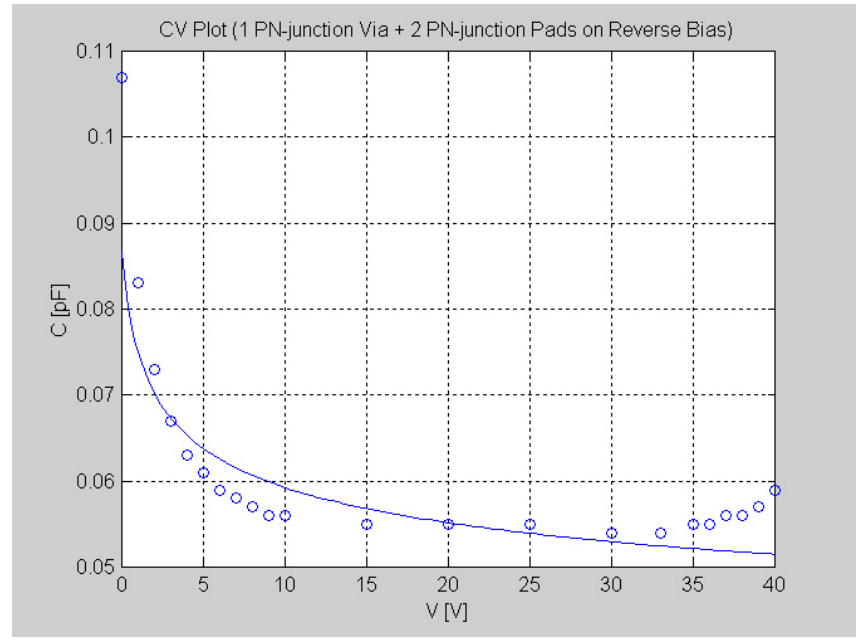

Figure 12. Comparison of capacitance-voltage relationships (CV) of a reversed biased pn junction through wafer interconnect.

\section{CONCLUSIONS}

The C-V measurements reveal that electrical through-wafer interconnects with parasitic capacitance of $0.05 \mathrm{pF}$ have been achieved. This makes possible of its application to more devices with lower capacitance. Although the series resistance of the through-wafer interconnects is not very serious for the CMUT application, it stands as a problem for the future. This series resistance can be substantially reduced by employing doped polysilicon or metal to seal the via holes which will be shown in a future work.

\section{ACKNOWLEDGEMENT}

This work is sponsored by the Office of Naval Research.

\section{REFERENCES}

1. C. H. Cheng, A. S. Ergun, B. T. Khuri-Yakub, " Electrical Through-Wafer Interconnects with Sub-PicoFarad Parasitic Capacitance," MEMS Conference 2001, Berkeley, California, 8/2426/2001.

2. C. H. Cheng, E. M. Chow, A. S. Ergun, and B. T. KhuriYakub, "An Efficient Electrical Addressing Method Using Through-Wafer Vias for Two-Dimensional Ultrasonic Arrays," Proceedings of 2000 IEEE International Ultrasonics Symposium, San Juan, Puerto Rico, 10/22-25/2000, pp. 1179-82.

3. B. T. Khuri-Yakub, C. H. Cheng, F. L. Degertekin, S. Ergun, S. Hansen, X. C. Jin, and O. Oralkan, "Silicon Micromachined Ultrasonic Transducers," presented at the Fifth European Conference on Underwater Acoustics, Lyon France, July 10-13, 2000 .

4. S. Calmes, C. H. Cheng, F. L. Degertekin, X. C. Jin, and B. T. Khuri-Yakub, "Highly Integrated 2-D Capacitive Micromachined Ultrasound Transducers," presented at the 1999 IEEE International Ultrasonics Symposium, Lake Tahoe, Nevada, Octobor 17-20, 1999; in Ultrasonics Symposium Proceedings, pp. 1163-6.

5. X. C. Jin, C. H. Cheng. O. Oralkan, S. Calmes, F. L. Degertekin, and B. T. Khuri-Yakub, "Recent Progress in Capacitive Micromachined Ultrasonic Immersion Transducer Array," presented at the 8th International Symposium on Integrated Circuits, Devices and Systems, Singapore, September 810, 1999; in ISIC-99 Proceedings, pp.159-162.

6. H. T. Soh, C. P. Yue, A. McCarthy, et al., "Ultra-Low Resistance, Through-Wafer Via (TWV) Technology and Its Application in Three Dimensional Structures on Silicon," Japanese Journal of Applied Physics, Part I 38(4B), 2393-6 (1999).

7. T. R. Anthony, "Forming Electrical Interconnections through Semiconductor Wafers," Journal of Applied Physics, 52(8) 5340-9 (1981).

8. E. M. Chow, A. Partridge, C. F. Quate, T. W. Kenny, "Through-Wafer Electrical Interconnects Compatible with Stanford Semiconductor Processing," Solid-State Sensor and Actuator Workshop, Hilton Head Island, South Carolina, June 4-8, 2000, pp. 343-6.

9. R. Rumpf, K. Suzuki, "Modeling of Irregular Boron Diffusion Profiles over Varied Surface Topologies," Digest of Papers. Microprocesses and Nanotechnology '99, pp. 198-9. 